\title{
Repair of Accidental DNA Double-Strand Breaks in the Human Genome and Its Relevance to Vector DNA Integration
}

\author{
Noritaka Adachi ${ }^{1,2, *}$, Shinta Saito ${ }^{1}$ and Aya Kurosawa ${ }^{1}$ \\ ${ }^{1}$ Graduate School of Nanobioscience, Yokohama City University, Yokohama 236-0027, Japan \\ ${ }^{2}$ Advanced Medical Research Center, Yokohama City University, Yokohama 236-0004, Japan
}

\section{HR and NHEJ: Two Major Pathways for Repair of Chromosomal DNA Double-Strand Breaks}

Efficient repair of chromosomal DNA damage is crucial for cells to maintain genome integrity. DNA double-strand breaks (DSBs) are the most severe type of DNA lesions that can be caused by various exogenous and endogenous mechanisms, such as ionizing radiation, reactive oxygen species, topoisomerase poisons, or replication errors [1]. DSBs, if left unrepaired or mis-repaired, lead to cell death or chromosomal aberrations [2,3]. Human cells have evolved two fundamentally different mechanisms for repairing chromosomal DSBs, homologous recombination (HR) and non-homologous end-joining (NHEJ) [4]. NHEJ not only repairs accidental (non-physiological) DSBs, but is also essential for rejoining physiological DSBs that arise in the process of $\mathrm{V}(\mathrm{D}) \mathrm{J}$ recombination in $\mathrm{B}$ and $\mathrm{T}$ lymphocytes and class switch recombination in mature B cells [3].

A wide variety of proteins have been identified thus far that contribute to the HR and NHEJ machineries [5]. HR is a highly complicated process of DNA transaction, in which Rad51 protein plays an essential role in DNA strand exchange with the aid of several other proteins such as Rad54, Brca2, Rad52, and Rad51 paralogs [6,7]. For HR to occur, DSBs should be processed (i.e., end-resected) to produce a long 3'-overhang single-stranded DNA [8,9], and recent studies have identified a number of proteins involved in end resection or its regulation; among these, Mre11 and CtIP play essential roles in the initial step of end resection [9-11]. In contrast to HR, NHEJ is thought to be a rather simpler process that requires, at least biochemically, only four proteins (two protein complexes); specifically, $\mathrm{Ku}$, a heterodimer of Ku70 and Ku80, initiates an NHEJ reaction by binding to the ends of a DSB, and the DNA ligase complex composed of Xrcc4 and Ligase IV (Lig4) seals the ends to complete repair [3]. In most cases, however, many other proteins do participate in NHEJ-mediated repair to trim the DSB ends, which are typically non-ligatable or non-compatible. These additional NHEJ factors involve DNA-PKcs, Artemis, XLF, and DNA polymerase $\mu / \lambda$; DNA-PKcs and Artemis have evolved in higher eukaryotes and do not exist in yeasts $[3,12]$. In addition to the classical pathway of NHEJ, recent evidence indicates the existence of a more error-prone mechanism of NHEJ called alternative endjoining that plays a role in DSB repair $[3,13]$. Alternative end-joining is $\mathrm{Ku} / \mathrm{Lig} 4$ independent and the precise mechanism remains largely unclear, although PARP1, Ligase III, and several factors involved in end resection (to initiate HR) have been implicated in DSB repair via alternative end-joining [14-16].

Which DSB repair pathway is beneficial for cells to preserve genome integrity? NHEJ (the classical NHEJ pathway) repairs broken DNA ends with little or no homology and is often associated with nucleotide loss, whereas HR allows for accurate repair of DSBs with the use of homologous DNA sequence, usually located on a sister chromatid $[3,4,12]$. Such difference in accuracy between the two pathways, however, does not mean that HR is superior to NHEJ in maintaining integrity of human genomes, which contain lots of repetitive DNA sequences [4]. For example, an HR reaction between Alu sequences in a cell would cause deleterious consequences and hence must be prohibited $[17,18]$. Thus, human somatic cells preferentially use NHEJ to repair accidental DSBs; in particular, in G0/G1 phase of the cell cycle, DSB repair is only performed by NHEJ, and HR is inert. Both NHEJ and HR can work, however, in S to G2 phases when DNA replication has been completed and the sister chromatid is available [19]. Thus, how and which pathway is chosen for repair of a $\operatorname{DSB}(s)$ has been a critical issue in the DNA repair field, and there has been a debate [4]. Recent evidence suggests that Ku-bound DSBs, where end resection does not occur, are directed to NHEJ, while end-resected DSBs, to which Ku cannot bind, are channeled to HR (or alternative end-joining) [20-24]. Thus, in addition to the end binding protein $\mathrm{Ku}$, various factors that regulate end resection are involved in DSB repair pathway choice [16,25-30]. Apparently, the type of DSB is also a determinant of pathway choice [31,32]; for example, replication-associated one-ended DSBs are preferentially repaired by HR, while topoisomerase II-mediated DSBs are almost exclusively repaired by NHEJ [33,34]. Interestingly, however, it appears that cells do not always choose a proper pathway to deal with induced DSBs. In fact, absence of NHEJ gives a growth advantage to cells accumulating replication-associated DSBs [34,35], although this may simply reflect the fact that NHEJ is basically the first choice to repair any type of those DSBs that naturally allow Ku-binding [4].

\section{Impact of DSB Repair Deficiency on Targeted and Random Integration}

Gene targeting via HR provides the definitive tool in analyzing gene function. For gene targeting to be successfully achieved, the target genome sequence should be replaced with the vector DNA (i.e., targeting vector), not with the sister chromatid. The principal limitation of conventional gene-targeting technology is the extremely low efficiency of HR-mediated targeted integration, which occurs at least 2-3 orders of magnitude less frequently than random integration [36], as depicted in Figure 1A.

Random integration is a phenomenon in which a transfected DNA molecule(s) are inserted into (random sites of) the host genome via non-homologous recombination. It has been generally assumed that random integration results from the repair of spontaneous chromosomal DSBs caused by endogenous factors. Indeed, we have recently shown that DNA topoisomerase II $a$ and reactive oxygen species (ROS) are such endogenous factors responsible for causing DNA damage that leads to random integration of transfected DNA in human cells [37]. Transient inhibition of topoisomerase IIa significantly increases

*Corresponding author: Noritaka Adachi, Graduate School of Nanobioscience, Yokohama City University, Yokohama 236-0027, Japan; Tel: 81-45-787-2228, Fax: 81-45-787-2228, E-mail: nadachi@yokohama-cu.ac.jp

Received December 26, 2013; Accepted December 27, 2013; Published December 30, 2013

Citation: Adachi N, Saito S, Kurosawa A (2013) Repair of Accidental DNA DoubleStrand Breaks in the Human Genome and Its Relevance to Vector DNA Integration. Gene Technology 3: e107. doi: 10.4172/2329-6682.1000e107

Copyright: ( 2013 Adachi N, et al. This is an open-access article distributed under the terms of the Creative Commons Attribution License, which permits unrestricted use, distribution, and reproduction in any medium, provided the original author and source are credited. 
A

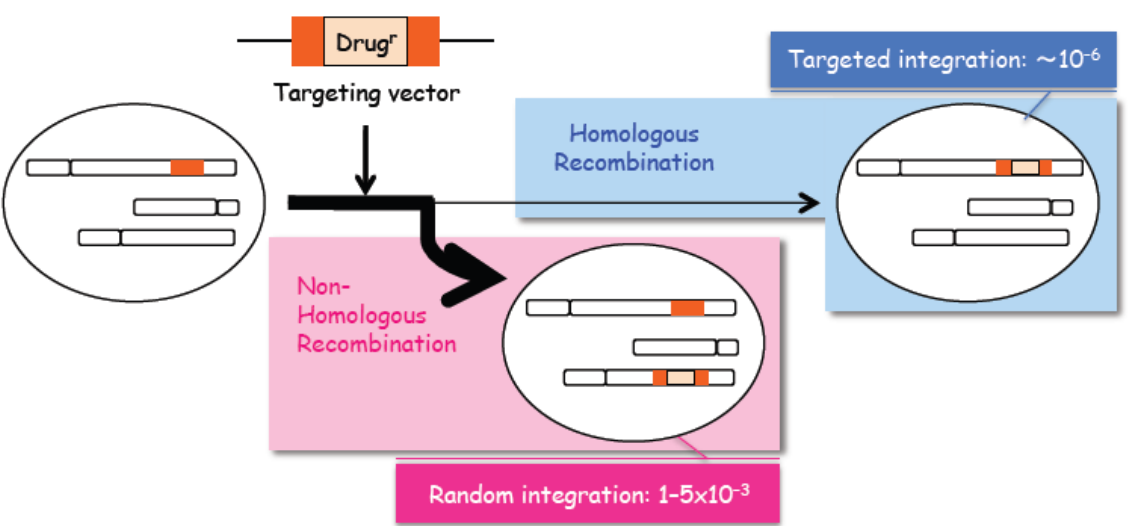

B

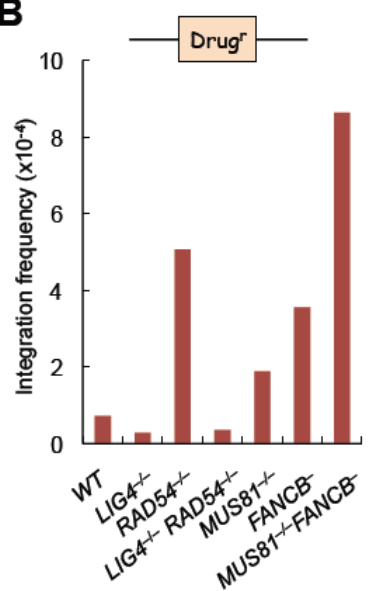

C

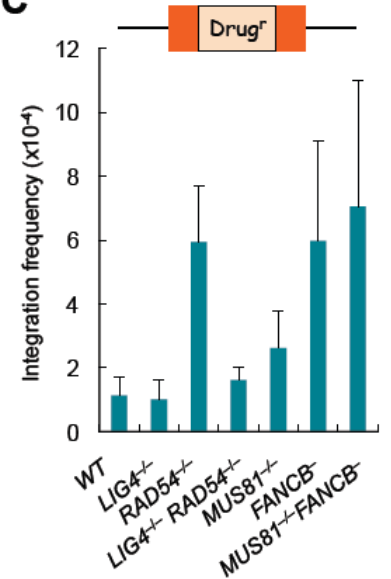

D

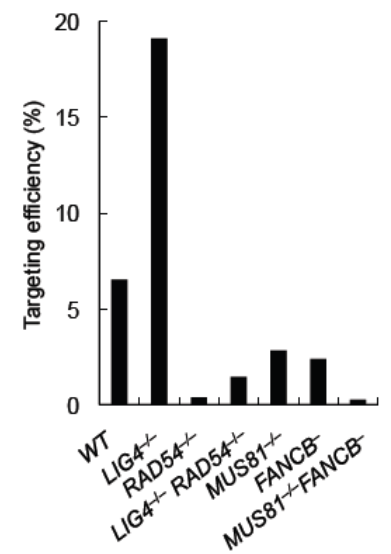

Figure 1: Impact of DSB repair deficiency on random and targeted integration.

(A) Gene targeting is quite inefficient in human somatic cells. When targeting vector is transfected into cells, random integration occurs at least 2 to 3 orders of magnitude more frequently than targeted integration. (B) Integration frequency of pßactin-His in human Nalm-6 cell lines. The DSB repair mutants $\left(L / G 4^{-1-}, R A D 54^{-1-}, L / G 4^{-1-}\right.$

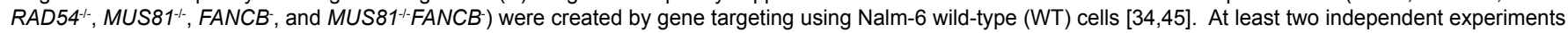
were performed for each cell line. Note that $\mathrm{p} \beta$ actin-His harbors little or no homology to the human genome [40]. (C, D) Integration frequency (C) and gene-targeting efficiency (D) of pHPRT-Hyg in the Nalm-6 cell lines. At least three independent experiments were performed for each cell line. The lengths of 5 ' and 3 ' arms of this targeting vector are 3.8 and $5.1 \mathrm{~kb}$, respectively [40].

random integration [38]; conversely, siRNA-mediated knockdown of topoisomerase IIa reduces random integration [37]. Cells continuously cultured under 3\% oxygen conditions after DNA transfection display reduced random-integration frequency compared to that under $21 \%$ oxygen conditions [37], although the gene-targeting efficiency was little affected by the low-oxygen culture condition (our unpublished observations).

Loss of NHEJ in lower eukaryotes results in significantly reduced or no random integration events, and thus, as high as $100 \%$ gene-targeting efficiency can be achieved by inactivating NHEJ (for example, [39]). In human somatic cells, however, suppression of NHEJ does not result in decreased random-integration frequency, although the efficiency of gene targeting can be increased [40] (Figure 1B-D). These findings clearly indicate that NHEJ is not the sole mechanism of random integration in human somatic cells, and suggest the contribution of alternative end-joining to the residual random integration events by non-homologous recombination. Intriguingly, unlike vectors with no or shorter homology arms, integration frequency of targeting vectors with long homology arms was not affected by LIG4 deficiency [40] (Figure 1B, C; data not shown). It could be that in the absence of NHEJ, homology arms of the targeting vector served to prevent marker gene loss caused by large deletion (chew-back); however, as these homology arms contain a number of Alu elements, it is more likely that homology arms serve to trigger random integration in an NHEJ-independent fashion. Earlier studies using rodent cell lines, along with the fact that alternative end-joining favors micro-homologies, strongly support this idea $[13,41,42]$.

Elimination of the HR protein Rad54 resulted in significantly reduced gene-targeting efficiency in human cells (Figure 1D), a finding consistent with previous reports using rodent and avian cell mutants $[43,44]$. Intriguingly, random-integration frequency was more than fivefold higher in RAD54-null cells than in their wild-type counterparts, implying that the observed reduction of gene-targeting efficiency in the absence of Rad54 is due, at least in part, to an unexpectedly increased random-integration frequency. Similar observations were made with mutant cell lines deficient in MUS81 and/or FANCB, genes implicated in HR [45,46] (Figure 1B-D). It is also important to note that the increased random-integration frequency associated with HR deficiency was suppressed by an additional loss of NHEJ, and this suppression was less pronounced when targeting vectors were used (Figure 1B,C). These 
data further support the aforementioned idea that random-integration frequency is substantially influenced by homologous sequences present in the vector, and that these DNA sequences may serve to trigger NHEJindependent, homology-based random integration. Thus, effective suppression of this mechanism will be a promising approach to reduce random integration events after targeting-vector transfection.

Despite the rapid progress on artificial nucleases (i.e., ZFN, TALEN, or CRISPR-based system) and their effective applications to targeted gene inactivation in various species [47], HR-mediated gene targeting (knock-in as well as knockout) without the use of artificial nucleases still provides an indispensable technique that must be further developed in the context of human-derived cells, as artificial nucleases are capable of causing DNA lesions that lead to deleterious off-target mutations [48-50]. It is expected that deciphering the molecular mechanism of random integration in terms of vector DNA sequence and precise DSB repair mechanisms will help improve human somatic cell gene targeting, for example, by developing a targeting vector that is most suitable for reducing random integrants.

\section{References}

1. Lieber MR, Gu J, Lu H, Shimazaki N, Tsai AG (2010) Nonhomologous DNA end joining (NHEJ) and chromosomal translocations in humans. Subcell Biochem 50: $279-296$

2. O'Driscoll M, Jeggo PA (2006) The role of double-strand break repair - insights from human genetics. Nat Rev Genet 7: 45-54.

3. Lieber MR (2010) The mechanism of double-strand DNA break repair by the nonhomologous DNA end-joining pathway. Annu Rev Biochem 79: 181-211.

4. Brandsma I, van Gent DC (2012) Pathway choice in DNA double strand break repair: observations of a balancing act. Genome Integr 3: 9 .

5. Thompson LH (2012) Recognition, signaling, and repair of DNA double-strand breaks produced by ionizing radiation in mammalian cells: the molecular choreography. Mutat Res 751: 158-246.

6. Karpenshif $\mathrm{Y}$, Bernstein KA (2012) From yeast to mammals: recent advances in genetic control of homologous recombination. DNA Repair (Amst) 11: 781-788.

7. Krejci L, Altmannova V, Spirek M, Zhao X (2012) Homologous recombination and its regulation. Nucleic Acids Res 40: 5795-5818.

8. Ira G, Pellicioli A, Balijja A, Wang X, Fiorani S, et al. (2004) DNA end resection, homologous recombination and DNA damage checkpoint activation require CDK1. Nature 431: 1011-1017.

9. Mimitou EP, Symington LS (2009) Nucleases and helicases take center stage in homologous recombination. Trends Biochem Sci 34: 264-272.

10. Sartori AA, Lukas C, Coates J, Mistrik M, Fu S, et al. (2007) Human CtIP promotes DNA end resection. Nature 450: 509-514.

11. Shibata A, Moiani D, Arvai AS, Perry J, Harding SM, et al. (2013) DNA DoubleStrand Break Repair Pathway Choice Is Directed by Distinct MRE11 Nuclease Activities. Mol Cell, In Press.

12. Kurosawa A, Adachi N (2010) Functions and regulation of Artemis: a goddess in the maintenance of genome integrity. J Radiat Res 51: 503-509.

13. Decottignies A (2013) Alternative end-joining mechanisms: a historical perspective. Front Genet 4: 48

14. Robert I, Dantzer F, Reina-San-Martin B (2009) Parp1 facilitates alternative NHEJ, whereas Parp2 suppresses IgH/c-myc translocations during immunoglobulin class switch recombination. J Exp Med 206: 1047-1056.

15. Truong LN, Li Y, Shi LZ, Hwang PY, He J, et al. (2013) Microhomology-mediated End Joining and Homologous Recombination share the initial end resection step to repair DNA double-strand breaks in mammalian cells. Proc Natl Acad Sci U SA 110: 7720-7725.

16. Grabarz A, Guirouilh-Barbat J, Barascu A, Pennarun G, Genet D, et al. (2013) A role for BLM in double-strand break repair pathway choice: prevention of CtIP/ Mre11-mediated alternative nonhomologous end-joining. Cell Rep 5: 21-28.

17. Deininger PL, Batzer MA (1999) Alu repeats and human disease. Mol Genet Metab 67: 183-193.
18. Ade C, Roy-Engel AM, Deininger PL (2013) Alu elements: an intrinsic source of human genome instability. Curr Opin Virol 3: 639-645.

19. Takata M, Sasaki MS, Sonoda E, Morrison C, Hashimoto M, et al. (1998) Homologous recombination and non-homologous end-joining pathways of DNA double-strand break repair have overlapping roles in the maintenance of chromosomal integrity in vertebrate cells. EMBO J 17: 5497-5508.

20. Fattah F, Lee EH, Weisensel N, Wang Y, Lichter N, et al. (2010) Ku regulates the non-homologous end joining pathway choice of DNA double-strand break repair in human somatic cells. PLoS Genet 6: e1000855.

21. Shao Z, Davis AJ, Fattah KR, So S, Sun J, et al. (2012) Persistently bound Ku at DNA ends attenuates DNA end resection and homologous recombination. DNA Repair (Amst) 11: 310-316.

22. Sun J, Lee KJ, Davis AJ, Chen DJ (2012) Human Ku70/80 protein blocks exonuclease 1-mediated DNA resection in the presence of human Mre11 or Mre11/Rad50 protein complex. J Biol Chem 287: 4936-4945.

23. Granata M, Panigada D, Galati E, Lazzaro F, Pellicioli A, et al. (2013) To trim or not to trim: progression and control of DSB end resection. Cell Cycle 12: 1848-1860.

24. Ferretti LP, Lafranchi L, Sartori AA (2013) Controlling DNA-end resection: a new task for CDKs. Front Genet 4: 99.

25. Kaidi A, Weinert BT, Choudhary C, Jackson SP (2010) Human SIRT6 promotes DNA end resection through CtIP deacetylation. Science 329: 1348-1353.

26. Symington LS, Gautier J (2011) Double-strand break end resection and repair pathway choice. Annu Rev Genet 45: 247-271.

27. Daugaard M, Baude A, Fugger K, Povlsen LK, Beck H, et al. (2012) LEDGF (p75) promotes DNA-end resection and homologous recombination. Nat Struct Mol Biol 19: 803-810.

28. Escribano-Díaz C, Orthwein A, Fradet-Turcotte A, Xing M, Young JT, et al. (2013) A cell cycle-dependent regulatory circuit composed of 53BP1-RIF1 and BRCA1-CtIP controls DNA repair pathway choice. Mol Cell 49: 872-883.

29. Steger M, Murina O, Hühn D, Ferretti LP, Walser R, et al. (2013) Prolyl isomerase PIN1 regulates DNA double-strand break repair by counteracting DNA end resection. Mol Cell 50: 333-343.

30. Zhou Y, Paull TT (2013) DNA-dependent Protein Kinase regulates DNA end resection in concert with the Mre11-Rad50-Nbs1 (MRN) complex and AtaxiaTelangiectasia-Mutated (ATM). J Biol Chem, In Press.

31. Yajima H, Fujisawa $H$, Nakajima NI, Hirakawa $H$, Jeggo PA, et al. (2013) The complexity of DNA double strand breaks is a critical factor enhancing endresection. DNA Repair (Amst) 12: 936-946.

32. Schipler A, lliakis G (2013) DNA double-strand-break complexity levels and their possible contributions to the probability for error-prone processing and repair pathway choice. Nucleic Acids Res 41: 7589-7605

33. Adachi N, Suzuki H, liizumi S, Koyama H (2003) Hypersensitivity of nonhomologous DNA end-joining mutants to VP-16 and ICRF-193: implications for the repair of topoisomerase II-mediated DNA damage. J Biol Chem 278 : 35897-35902.

34. Kurosawa A, Saito S, So S, Hashimoto M, Iwabuchi K, et al. (2013) DNA ligase IV and artemis act cooperatively to suppress homologous recombination in human cells: implications for DNA double-strand break repair. PLoS One 8 : e72253.

35. Adachi N, So S, Koyama H (2004) Loss of nonhomologous end joining confers camptothecin resistance in DT40 cells. Implications for the repair of topoisomerase I-mediated DNA damage. J Biol Chem 279: 37343-37348.

36. Vasquez KM, Marburger K, Intody Z, Wilson JH (2001) Manipulating the mammalian genome by homologous recombination. Proc Natl Acad Sci U S A 98: 8403-8410

37. Kamekawa H, Kurosawa A, Umehara M, Toyoda E, Adachi N (2013) Endogenous factors causative of spontaneous DNA damage that leads to random integration in human cells. Gene Technology 2: 1000105.

38. Toyoda E, Kurosawa A, Kamekawa H, Adachi N (2009) Topoisomerase Ilalpha inhibition following DNA transfection greatly enhances random integration in a human pre-B lymphocyte cell line. Biochem Biophys Res Commun 382: 492 496.

39. Ninomiya $Y$, Suzuki K, Ishii C, Inoue H (2004) Highly efficient gene replacements in Neurospora strains deficient for nonhomologous end-joining. Proc Natl Acad Sci U S A 101: 12248-12253 
Citation: Adachi N, Saito S, Kurosawa A (2013) Repair of Accidental DNA Double-Strand Breaks in the Human Genome and Its Relevance to Vector DNA Integration. Gene Technology 3: e107. doi: 10.4172/2329-6682.1000e107

40. liizumi S, Kurosawa A, So S, Ishii Y, Chikaraishi Y, et al. (2008) Impact of nonhomologous end-joining deficiency on random and targeted DNA integration: implications for gene targeting. Nucleic Acids Res 36: 6333-6342.

41. Merrihew RV, Marburger K, Pennington SL, Roth DB, Wilson JH (1996) Highfrequency illegitimate integration of transfected DNA at preintegrated target sites in a mammalian genome. Mol Cell Biol 16: 10-18.

42. Liang F, Romanienko PJ, Weaver DT, Jeggo PA, Jasin M (1996) Chromosomal double-strand break repair in Ku80-deficient cells. Proc Natl Acad Sci U S A 93: 8929-8933

43. Bezzubova O, Silbergleit A, Yamaguchi-Iwai $Y$, Takeda S, Buerstedde JM (1997) Reduced X-ray resistance and homologous recombination frequencies in a RAD54-/- mutant of the chicken DT40 cell line. Cell 89: 185-193.

44. Essers J, Hendriks RW, Swagemakers SM, Troelstra C, de Wit J, et al (1997) Disruption of mouse RAD54 reduces ionizing radiation resistance and homologous recombination. Cell 89: 195-204.
45. Nomura $Y$, Adachi N, Koyama $H$ (2007) Human Mus81 and FANCB independently contribute to repair of DNA damage during replication. Genes Cells 12: 1111-1122.

46. Kikuchi K, Narita T, Pham VT, lijima J, Hirota K, et al. (2013) Structure-specific endonucleases xpf and mus81 play overlapping but essential roles in DNA repair by homologous recombination. Cancer Res 73: 4362-4371.

47. Gaj T, Gersbach CA, Barbas CF 3rd (2013) ZFN, TALEN, and CRISPR/Casbased methods for genome engineering. Trends Biotechnol 31: 397-405.

48. Grau J, Boch J, Posch S (2013) TALENoffer: genome-wide TALEN off-target prediction. Bioinformatics 29: 2931-2932.

49. Cho SW, Kim S, Kim Y, Kweon J, Kim HS, et al. (2013) Analysis of off-target effects of CRISPR/Cas-derived RNA-guided endonucleases and nickases. Genome Res, In Press.

50. Fu Y, Foden JA, Khayter C, Maeder ML, Reyon D, et al. (2013) High-frequency off-target mutagenesis induced by CRISPR-Cas nucleases in human cells. Nat Biotechnol 31: 822-826. 